\title{
300 Diagnostic value of low b value diffusion weighted MRI in patients with acute myocardial infarction
} Kunihiko Teraoka*1, Hajime Sakuma ${ }^{2}$, Masashi Kawade ${ }^{1}$, Shintaro Kiuchi ${ }^{1}$, Yoshinori Suzuki ${ }^{1}$, Yoshiaki Komori ${ }^{3}$, Masao Yamada ${ }^{1}$, Masaharu Hirano ${ }^{1}$, Kenji Takazawa ${ }^{1}$ and Akira Yamashina ${ }^{1}$

\author{
Address: ${ }^{1}$ Tokyo Med. University, Tokyo, Japan, ${ }^{2}$ Mie University Graduate School of Medicine, Tsu, Japan and ${ }^{3}$ Siemens Asahi Medical \\ Technologies Ltd, Tokyo, Japan \\ * Corresponding author
}

from I Ith Annual SCMR Scientific Sessions

Los Angeles, CA, USA. I-3 February 2008

Published: 22 October 2008

Journal of Cardiovascular Magnetic Resonance 2008, I O(Suppl I):AI03 doi:I0.I I86/I532-429X-I0-SI-AI 03

This abstract is available from: http://jcmr-online.com/content/I0/SI/AI03

(C) 2008 Teraoka et al; licensee BioMed Central Ltd.

\section{Introduction}

Diffusion-weighted MRI has been widely employed to detect early ischemic injury of the brain. Diffusionweighted MRI is potentially useful for detecting AMI.

\section{Purpose}

The purposes of this study were to evaluate the feasibility of diffusion weighted MR (DW-MR) Image of the myocardium in patients with acute myocardial infarction (AMI), to determine the optimal b-value to visualize AMI, and to compare DW-MR images with Late gadolinium enhanced MR (LGE-MR) Image.

\section{Methods}

Twenty AMI patients were prospectively enrolled. A clinical $1.5 \mathrm{~T}$ MR imager was used. The MRI was performed at a mean of $4.7 \pm 2.3$ days after the onset of AMI.

Free breathing diffusion weighted MR images were acquired with a respiratory and ECG gated single shot echo planar sequence with a number of averages of 5 , and b values of $0,50,100,150,200$ and $300 \mathrm{~s} / \mathrm{mm}^{2}$. LGE MR images were obtained 10 minutes after injection of 0.15 $\mathrm{mmol} / \mathrm{kg}$ of gadolinium contrast medium. In quantitative analysis, the averaged ADC was $4.6 \pm 2.2 \times 10^{-3} \mathrm{~mm}^{2} / \mathrm{s}$ for normal myocardium and $3.6 \pm 1.2 \times 10^{-3} \mathrm{~mm}^{2} / \mathrm{s}$ for infracted myocardium, being substantially higher than the ADC of water at body temperature. However, blood signal was effectively suppressed on low b value diffusion weighted MRI acquired with b value of $50 \mathrm{~s} / \mathrm{mm}^{2}$, permitting visual determination of endocardial border in the dysfunctional segments in patients with AMI.

The observers traced epicardial and endocardial borders of the left ventricular wall, and determined high intensity area on DW-MR image and LGE area on contrast enhanced MRI as a percentage of the total LV area.

\section{Results}

1) On low b value DW-MR images, high signal intensity was noted in all of 20 patients in the myocardial segment that corresponded to the myocardial territories perfused by the culprit coronary artery.

2) The ratio of signal intensity of high intensity area to remote normal area on low-b value DW-MRI, which was significantly higher than that of remote normal to another remote normal myocardium $(2.87 \pm 1.42$ vs $0.98 \pm 0.07$ $(\mathrm{P}<0.0001))$.

3) All infarct lesions detected by low b-value DW-MRI exhibited LGE contrast enhanced MRI.

4) High intensity area on DW-MRI was significantly larger than LGE area on contrast enhanced MRI. 5) Area of infarct-related high intensity on DW-MRI showed significant 
linear correlation with LGE area in 20 patients with AMI $(\mathrm{r}=0.825(\mathrm{P}<0.0001))$

\section{Conclusion}

AMI can be accurately detected with low b-value DW-MR I. Diffusion sensitive gradients effectively suppressed the confusing bright signal from blood pool in the left ventricular chamber. Imaging approach that combines low bvalue DW-MRI and LGE-MRI seems to be promising for the prediction of functional recovery in patients with AMI.

Publish with Bio Med Central and every scientist can read your work free of charge

"BioMed Central will be the most significant development for disseminating the results of biomedical research in our lifetime. " Sir Paul Nurse, Cancer Research UK

Your research papers will be:

- available free of charge to the entire biomedical community

- peer reviewed and published immediately upon acceptance

- cited in PubMed and archived on PubMed Central

- yours - you keep the copyright 